\title{
A CLASSIFICATION TREE TO IDENTIFY FACTORS AFFECTING TEMPOROMANDIBULAR DISORDERS
}

\author{
Ayse OZCAN-KUCUK ${ }^{1}$, Utku Nezih YILMAZ², Bilal EGE ${ }^{3}$, Mahmut KOPARAL ${ }^{3}$, Eda Didem YALÇIN ${ }^{4}$, Fatih \\ UCKARDES ${ }^{5 *}$
}

${ }^{1}$ Department of Oral and Maxillofacial Surgery, Faculty of Dentistry, Mersin University, 33190, Mersin, Turkey

${ }^{2}$ Department of Oral and Maxillofacial Surgery, Faculty of Dentistry, Dicle University, 21280, Diyarbakır, Turkey

${ }^{3}$ Department of Oral and Maxillofacial Surgery, Faculty of Dentistry, Adlyaman University, 02040, Adıyaman, Turkey

${ }^{4}$ Department of Dentomaxillofacial Radiology, Faculty of Dentistry, Gaziantep University, 27410, Sehitkamil, Gaziantep, Turkey

${ }^{5}$ Department of Biostatistics and Medical Informatics, Adlyaman University, Research and Education Hospital, 02040, Adlyaman, Turkey

\begin{abstract}
To examine the effects of stress, bruxism, malocclusion, and oral habits on the incidence of temporomandibular disorders (TMDs) using a classification tree method (CTM). We obtained data from a total of 1.338 patients: 669 who had TMDs and 669 who but haven't TMDs. We recorded interview data on stress and oral habits, and during oral examination, noted malocclusions, bruxism, and any sign of poor oral habits. All data were subjected to CTM analyses. The TMD rate was highest in stressed patients (96.8\%), and significantly lower in stress-free patients $(25.3 \%)(\mathrm{P}<0.001)$. TMD was evident in all patients exhibiting both stress and bruxism (100.0\%). In stressed patients lacking bruxism, the effects of malocclusion on TMD were significant $(\mathrm{P}<0.001)$. CTM analyses showed that the most significant factor affecting TMD was stress, followed by bruxism and malocclusion. This is the first report to use CTM to define factors affecting TMD.
\end{abstract}

Keywords: Bruxism, Classification tree method, Data mining, Stress, Temporomandibular disorders

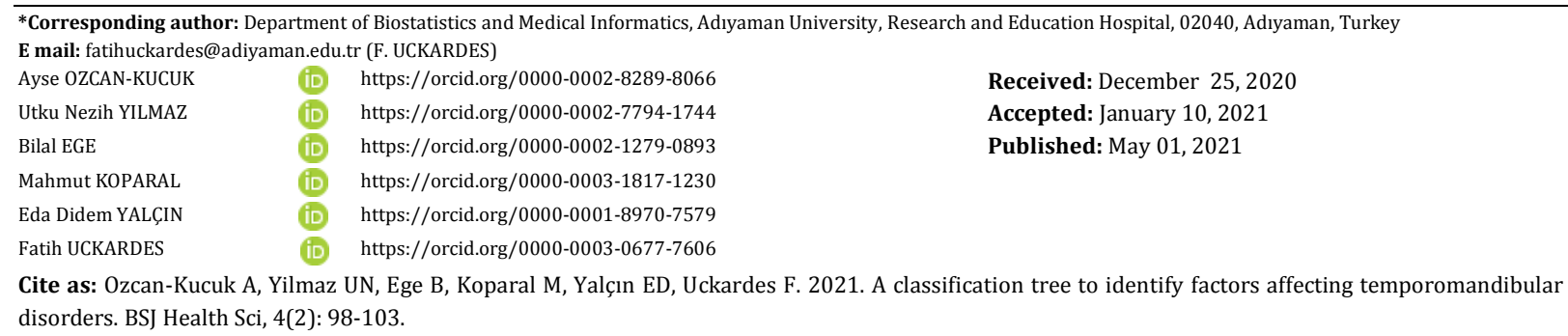

\section{Introduction}

Temporomandibular disorders (TMDs) include conditions of the masticatory muscles, the temporomandibular joint (TMJ), or both, and constitute a subgroup of musculoskeletal disorders (Scrivani et al., 2008). The most important signs and symptoms of TMD are joint pain during mandibular function, restricted mandibular movement, limited mouth-opening, jawlocking when the mouth is open or closed, and a noisy TMJ during jaw movement (Mujakperuo et al., 2010; Leite et al., 2013). Traditionally, these disorders have been the concerns of dentists. Several epidemiological studies on the prevalence of TMD in children and adolescents have been published, and rates of 9.8-80\% have been reported. However, the prevalence in Turkey remains poorly known. Approximately $75 \%$ of the general population exhibits at least one sign of TMD and approximately $33 \%$ has at least one symptom (List et al., 1999; Luther et al., 2016), but only $25 \%$ are aware of the symptoms and signs and report them to specialists (List et al., 1999).
The aetiology of TMD is both complex and multifactorial. Many factors including bruxism and other parafunctional habits, occlusal abnormalities, psychological features, and biological parameters can contribute to its development (Al-Ani et al., 2004; Chisnoiu et al., 2015). However, the detailed aetiology of the condition remains unknown. Therefore, TMD is difficult to diagnose and manage. Successful management requires identification and control of risk factors. An understanding of TMD aetiology is extremely important in this context.

Oral parafunctional habits are generally considered to contribute to the aetiology of TMDs and have been studied in children and adults. Tooth-clenching and grinding (bruxism), nail-biting, and gum-chewing are the most common such factors reported (Michelotti et al., 2010). Epidemiological studies have found associations between oral parafunctions and TMD signs and symptoms. Widmalm et al. (1995) reported that parafunctional habits, particularly bruxism, may be risk factors for TMD. However, few other studies have found any statistically significant associations between 
parafunctional habits and TMDs (Castelo et al., 2005; van der Meulen et al., 2006). Over the past 20 years, many studies have investigated the relationship between stress and TMD (Kanehira et al., 2008; Mottaghi et al., 2011). Stress is significantly associated with TMD symptoms and parafunctionality (Kanehira et al., 2008). Matsuka et al. (2008) compared factors contributing to TMD and concluded that psychological features were the most important. Deleeuw and Bertoli (2005) found that stress were significantly associated with the incidence of TMD.

Occlusion is also a major aetiological factor; different types of malocclusion are associated with various TMD signs and symptoms. The most common such factors are a large overjet, an anterior open bite, a posterior crossbite, and Angle Class II or III dental relationships. An extensive systematic review reported significant associations between the presence of some occlusal factors (a skeletal open bite, a unilateral crossbite, the absence of five or more teeth, a deep overbite, and a severe overjet) and TMD signs and symptoms (McNamara Jr et al., 1995). Tagkli et al. (2017) reported that patients with normal occlusion had fewer TMD symptoms than those with any type of malocclusion, either treated or untreated.

Data mining (often termed knowledge discovery) is a powerful statistical method; a large dataset gathered from different perspectives can be summarised and analysed to yield useful practical information revealing important relationships (Kantardzic, 2008). Technically, data mining features the discovery of correlations or patterns among dozens of fields in large relational databases (Ito et al., 2011). Classification and Regression Tree (CART) analysis, a data mining technique, is innovative, powerful, and of significant clinical utility. CART analyses use a decision tree to classify data. The trees are clear and easy to interpret. Thus, they are widely used in medicine (D'Alisa et al., 2006; Barlin et al., 2013; Patel et al., 2014; Zimmerman et al., 2016), biology (Vayssières et al., 2000), and psychology (Rosenfeld and Lewis, 2005). They have recently been used in dentistry (Ito et al., 2011; Arino et al., 2016; Machuca et al., 2018). Here, we employed the classification tree method (CTM) to analyse aetiological factors in patients with TMDs. We also sought interactions among such factors. The results can be used to identify those at higher risk for TMD.

\section{Material and Methods}

We obtained data from a total of 1.338 patients: 669 had TMDs and 669 received routine treatment for dental problems and did not have TMDs. Each participant underwent a full TMD examination. Interviews explored stress and oral habits (bruxism, biting of foreign objects, nail-biting, bottle-opening with the teeth, and gumchewing). In intraoral examinations, tooth wear, soft tissue signs of bruxism (linea alba), and tongue indentations were noted to establish whether parafunctional activities were associated with the anamnestic and clinical findings of TMD. In addition, malocclusion was noted as present or absent. All patients were aged 18-60 years old. The exclusion criteria were an age outside this range, receipt of TMD treatment elsewhere, receipt of orthodontic treatment, edentulous status, any central or peripheral neurological disorder, and/or a history of trauma.

CTM examines discrete dependent variables and the relationships between independent and dependent variables (Yamauchi et al., 2001; Chang et al., 2006) represented by visual nodes (Hebert et al., 2006). The initial node, termed the root node, is the most heterogeneous. The following nodes (the child nodes) are more homogenous, and the terminal node is the most homogenous (Camdeviren et al., 2007). The root node includes all dependent and independent variables. The aim is to homogeneously separate the terminal node from the child nodes at the highest level, and to exclude variables that are not related to the dependent variables. Such processing is termed splitting. We used the CTM to evaluate the effects of independent variables (malocclusion, bruxism, parafunction, and stress) on TMD and interactions among the factors.

The growth method featured chi-square automatic interaction detection (CHAID). This identifies the independent (predictor) variable exhibiting the strongest interaction with the dependent variable. The algorithm selects a set of predictors, examines their interactions, and predicts the optimal value of the dependent variable, eventually creating a classification tree. All statistical analyses were performed using SPSS ver. 15.0 for Windows. A P-value $<0.05$ was considered statistically significant.

\subsection{Ethical Consideration}

This study was approved by the Human Ethics Committee (Approval number: 218/3-13) and was performed in accordance with the Declaration of Helsinki.

\section{Results}

Table 1 lists subject age and sex and the incidences of stress, psychological problems, malocclusion, bruxism, and poor oral habits. TMD incidence was not associated with age, sex, or any psychological problems (all $\mathrm{P}>$ 0.05). Thus, these factors were not included in the decision tree. The optimum tree is shown in Figure 1; it predicts TMD by reference to stress, bruxism, malocclusion, and poor oral habits. Node 0 (the root node) contains descriptive TMD statistics, and was the most heterogeneous node (Figure 1). Stress, malocclusion, bruxism, and poor oral habits were significantly associated with the incidence of TMD (all P < 0.001); stress was the most significant factor. Thus, stress constituted the terminal node. The other factors formed child nodes in which stress was present or absent. TMD was most prominent in stressed patients (Node $1 ; 96.8 \%$ ), and significantly less common in those without stress (Node 2; 25.3\%) $(\mathrm{P}<0.001)$. 
Black Sea Journal of Health Science

Table 1. Descriptive statistics and the effects of various factors on temporomandibular disorders

\begin{tabular}{|c|c|c|c|c|}
\hline \multirow[b]{5}{*}{ Aetiological factor } & \multicolumn{4}{|c|}{ Group } \\
\hline & \multicolumn{2}{|c|}{ TMD } & \multicolumn{2}{|c|}{ Control } \\
\hline & $\mathrm{n}$ & $\%$ & $\mathrm{n}$ & $\%$ \\
\hline & 669 & 50 & 669 & 50 \\
\hline & \multicolumn{2}{|c|}{ Yes } & \multicolumn{2}{|c|}{ No } \\
\hline Poor oral habits & 472 & 35.3 & 866 & 64.7 \\
\hline Malocclusion & 532 & 39.8 & 806 & 60.2 \\
\hline Psychological problems & 168 & 12.6 & 1170 & 87.4 \\
\hline Bruxism & 294 & 22.0 & 1044 & 78 \\
\hline \multirow[t]{2}{*}{ Stress } & 462 & 34.5 & 876 & 65.5 \\
\hline & Mean & SD & Mean & SD \\
\hline Age & 33.23 & 12.789 & 33.91 & 13.082 \\
\hline \multirow[t]{3}{*}{ Sex } & \multicolumn{2}{|c|}{ Female } & \multicolumn{2}{|c|}{ Male } \\
\hline & $\mathrm{n}$ & & $\mathrm{n}$ & \\
\hline & \multicolumn{2}{|l|}{724} & 614 & 45.9 \\
\hline
\end{tabular}

$\mathrm{TMD}=$ temporomandibular disorders, $\mathrm{SD}=$ standard deviation.

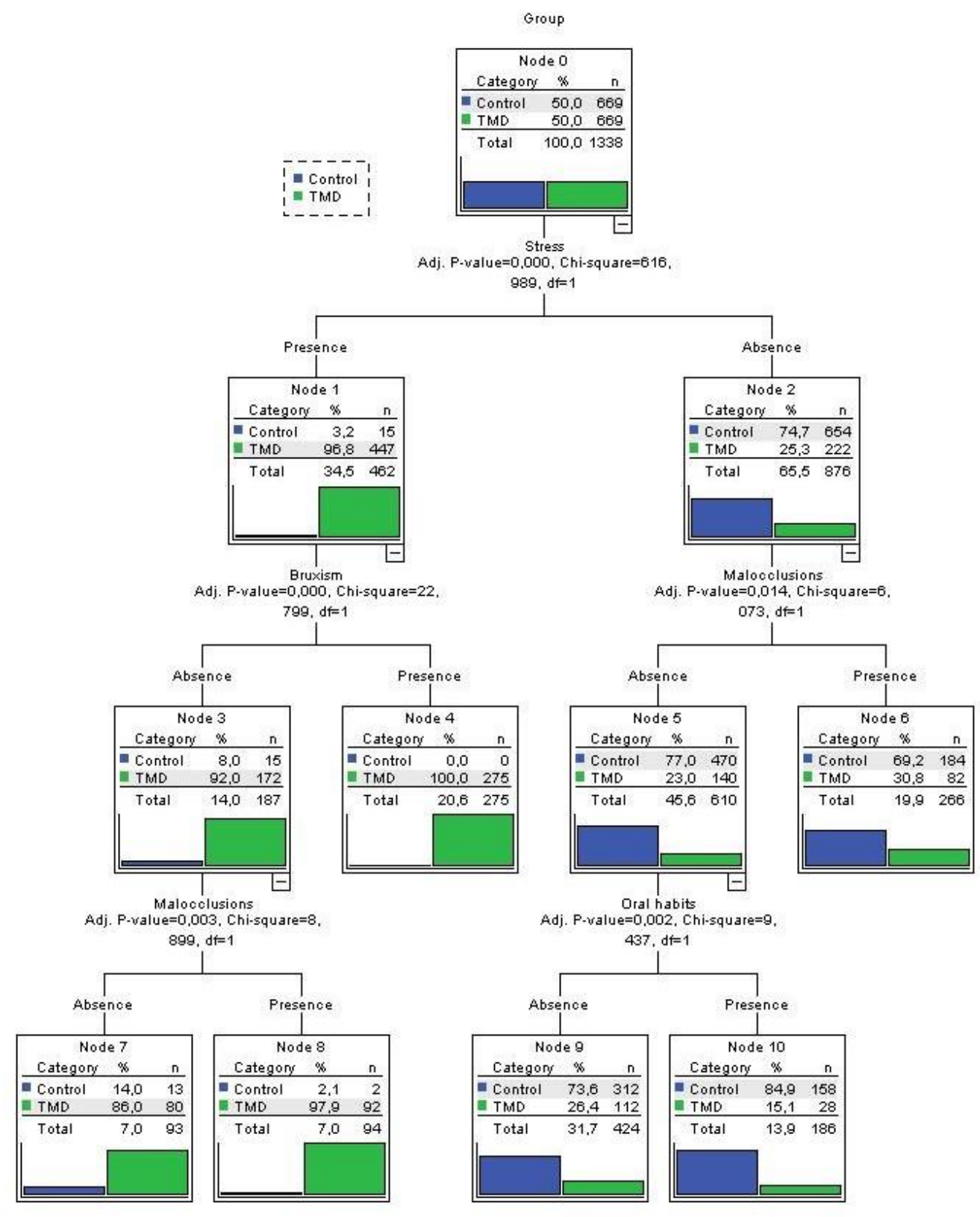

Figure 1. Optimal regression tree. 
TMD was detected in all patients with both stress and bruxism (Node 4; 100.0\%), but was significantly less prevalent in stressed patients without bruxism (92.0\%) $(\mathrm{P}<0.001)$. In patients who were stressed and did not exhibit bruxism, the effects of malocclusion on TMD were significant $(\mathrm{P}<0.001)$. TMD was detected in $97.9 \%$ of patients with malocclusion (Node 8) and in $86.0 \%$ without malocclusion (Node 7), a significant difference ( $P$ $<0.01$ ).

Malocclusion and poor oral habits were associated with a higher rate of TMD in non-stressed patients $(\mathrm{P}<0.001)$. TMD incidence was higher in stress-free patients with than without malocclusions (Node 6, 30.8\%; Node 5, $23.0 \%$, respectively) $(\mathrm{P}<0.05)$. Poor oral habits were associated with a higher incidence of TMD in patients without stress but with malocclusion ( $<<0.001)$. TMD was detected in $15.1 \%$ of patients with poor oral habits (Node 10), being more common in such patients than in those without such habits (Node 9; 26.4\%).

We used CHAID to grow the tree. We employed risk and classification data to evaluate model performance. The risk estimate was 0.177, indicating that the category predicted by the model was wrong in $17.7 \%$ of cases. Thus the model classified approximately $82.3 \%$ of TMD cases correctly. Thus, $82.3 \%$ of the variation in TMD is explained by the independent variables that had significant effects on tree growth.

\section{Discussion}

It is essential to understand the aetiology of TMD to identify and manage pathological factors. Stress, bruxism, malocclusion, oral habits, age, sex, and physiological problems are among possible TMD predictors. Four factors were significantly associated with TMD; these were stress, bruxism, malocclusion, and poor oral habits. CTM is not widely used in dentistry but finds many applications in biology and medicine. We use this method to identify the most important aetiological factors of TMD.

The effects of stress on TMD were more significant than those of bruxism, malocclusion, or poor oral habits; stress lay nearest to the root node (and was thus the terminal node). This node was rather homogenous in structure and could be separated into two different nodes (1 and 2 ). The highest TMD rate $(96.8 \%)$ was evident in stressed patients (Node 1), being only $25.3 \%$ in stress-free patients (Node 2). Stress has been considered important in terms of TMD onset. Many studies have evaluated the importance of stress in this context; some have found relevant associations (Vanderas and Papagiannoulis, 2002; Uhac et al., 2003; Deleeuw and Bertoli, 2005; Kanehira et al., 2008). List et al. (1999) found that psychosocial factors, such as increased stress, played more prominent roles than dental factors in adolescents with TMD. Filho et al. (2007) concluded that stress can strongly predispose one to TMD and inhibit recovery.

Oral parafunctional habits involve the mouth, tongue, and jaw, and may include bruxism, nail-biting, gum-chewing, pencil- or pen-chewing, tongue-thrusting, and thumbsucking. Of these, bruxism has been considered significant in terms of TMD development (Gavish et al., 2000; Sato et al., 2006). Michelotti et al. (2010) found an association between tooth-clenching/grinding and disc displacement; daytime bruxism was significantly more common among TMD patients than controls. Melchior found that bruxism, but not gum-chewing or onychophagia, affected TMD progression and/or nonremission (Melchior et al., 2012). Cortese and Biondi reported that various dysfunctions and parafunctions were significantly associated with TMD symptoms (Cortese and Biondi, 2009). We found that bruxism significantly affected the incidence of TMD, which was 92\% in stressed patients lacking bruxism (Node 3). TMD was present in all patients exhibiting both stress and bruxism (Node $4 ; 100 \%$ ). In addition, poor oral habits were associated with a higher incidence of TMD in stressfree patients without malocclusion. However, TMD was detected in $15.1 \%$ of patients who engaged in nail-biting, gum-chewing, or biting of foreign objects.

The aetiological importance of certain occlusal factors in terms of TMD development has been studied (Pullinger and Seligman, 2000; Egermark et al., 2003), but the results are controversial. An extensive systematic review, concluded that significant associations were evident between certain occlusal factors (a skeletal open bite, a unilateral crossbite, the absence of five or more teeth, a deep overbite, and a severe overjet) and TMD signs and symptoms (McNamara Jr et al., 1995). Pullinger et al. (1993) found only a weak correlation between occlusion and TMD; the incidence of TMD was affected only slightly by occlusal factors such as an open bite, an overjet $>6-7$ $\mathrm{mm}$, contact/intercuspal retrusion with sliding $>4 \mathrm{~mm}$, a unilateral lingual cross-bite, five or more missing posterior teeth, faulty restorations, and ill-fitting prostheses. Seven years later, the same researchers estimated that occlusal factors contributed about 10$20 \%$ to the total spectrum of aetiological factors differentiating healthy persons from those with TMD (Pullinger and Seligman, 2000). We found that malocclusion significantly affected TMD. The TMD rate (30.8\%) was higher in stress-free patients with than without malocclusions; $97.9 \%$ of stressed patients without bruxism had malocclusion and TMD.

We examined the effects of various factors on TMD and interactions among the factors. Stress was more important than bruxism, malocclusion, or oral habits. CTM can be used when several factors must be examined together, particularly if the dataset is large.

\section{Author Contributions}

AOK: data collection or management, manuscript writing and language editing, UNY: data collection or management, BE; data collection or management, MK: data collection or management and consultant, EDY: data collection or management, FU: statistical analysis, 
language editing and corresponding author.

\section{Conflict of Interest}

The authors declare that there is no conflict of interest.

\section{References}

Al-Ani MZ, Davies SJ, Gray RJM, Sloan P, Glenny AM. 2004. Stabilisation splint therapy for temporomandibular pain dysfunction syndrome. Cochrane Database Syst Rev, 1: CD002778.

Arino M, Ito A, Fujiki S, Sugiyama S, Hayashi M. 2016. Multicenter study on caries risk assessment in adults using survival classification and regression trees. Sci Rep, 6: 29190.

Barlin JN, Zhou Q, St Clair CM, Iasonos A, Soslow RA, Alektiar KM, Hensley ML, Jr. Leitao MM, Barakat RR, Abu-Rustum NR. 2013. Classification and regression tree (CART) analysis of endometrial carcinoma: Seeing the forest for the trees. Gynecol Oncol, 130: 452-456.

Camdeviren HA, Yazici AC, Akkus Z, Bugdayci R, Sungur MA. 2007. Comparison of logistic regression model and classification tree: An application to postpartum depression data. Expert Sys App, 32: 987-994.

Castelo PM, Gaviao MB, Pereira LJ, Bonjardim LR. 2005. Relationship between oral parafunctional/nutritive sucking habits and temporomandibular joint dysfunction in primary dentition. Int J Paediatr Dent, 15: 29-36.

Chang LY, Wang HW. 2006. Analysis of traffic injury severity: An application of non-parametric classification tree techniques. Acc Anly Prev, 38: 1019-1027.

Chisnoiu AM, Picos AM, Popa S, Chisnoiu DP, Lascu L, Picos A, Chisnoiu R. 2015. Factors involved in the etiology of temporomandibular disorders: A literature review. Clujul Med, 88: 473-478.

Cortese SG, Biondi AM. 2009. Relationship between dysfunctions and parafunctional oral habits, and temporomandibular disorders in children and teenagers. Arch Argent Pediatr, 107: 134-138.

D’Alisa S, Miscio G, Baudo S, Simone A, Tesio L, Mauro A. 2006. Depression is the main determinant of quality of life in multiple sclerosis: A classification regression (CART) study. Disabil Rehabil, 28: 307-314.

Deleeuw R, Bertoli E. 2005. Prevalence of traumatic stressor in patient with temporomandibular disorders. J Oral Maxillofac Surg, 63: 42-50.

Egermark I, Magnusson T, Carlsson GE. 2003. A 20-year followup of signs and symptoms of temporomandibular disorders and malocclusions in subjects with and without orthodontic treatment in childhood. Angle Orthod, 73: 109-115.

Filho J, Manzi FR, de Freitas DQ, Bóscolo NF, de Almeida MS. 2007; Evaluation of temporomandibular joint in stress-free patients. Dentomaxillofac Radiol, 36: 336-340.

Flannery B, Wisniewski SR. 2016. Classification and Regression Tree [CART] analysis to predict influenza in primary care patients. BMC Infect Dis, 16: 503-513.

Fonarow GC, Adams KFJr, Abraham WT, Yancy CW, Boscardin WJ. 2005. Risk stratification for in-hospital mortality in acutely decompensated heart failure: Classification and regression tree analysis. JAMA, 293: 572-580.

Gavish A, Halachmi M, Winocur E, Gazit E.2000. Oral habits and their association with signs and symptoms of temporomandibular disorders in adolescent girls. J Oral Rehabil, 27: 22-32.

Hebert M, Collin-Vezina D, Daigneault I, Parent N, Tremblay C. 2006. Factors linked to outcomes in sexually abused girls: a regression tree analysis. Comp Psych, 47: 443-455.

Ito A, Hayashi M, Hamasaki T, Ebisu S. 2011. Risk assessment of dental caries by using classification and regression trees. J Dent, 39: 457-463.

Kanehira H, Agariguchi A, Kato H, Pozveh ZE, Jahangirmoghaddam M. 2008. Association between stress and temporomandibular disorder. Nihon Hotetsu Shika Gakkai Zasshi, 52: 375-380.

Kantardzic M. 2003. Data mining: Concepts, models, methods, and algorithms, 2nd ed. John Wiley \& Sons, NewYork, USA.

Leite RA, Rodrigues JF, Sakima MT, Sakima T. 2013. Relationship between temporomandibular disorders and orthodontic treatment: A literature review. Dental Press J Orthod, 18: 150-157.

List T, Stenstrom B, Lundstrom I, Dworkin FS. 1999. TMD in patients with primary Sjogren syndrome: A comparison with temporomandibular clinic cases and controls. J Orofac Pain, 13: 21-28.

List T, Wahlund K, Larsson B. 2001. Psychosocial functioning and dental factors in adolescents with temporomandibular disorders: A case-control study. J Orofac Pain, 15: 218-227.

Luther F, Layton S, McDonald F. 2016. Orthodontics for treating temporomandibular joint (TMJ) disorders. Cochrane Database Syst Rev, 7: CD006541.

Machuca C, Vettore MV, Krasuska M, Baker SR, Robinson PG. 2017. Using classification and regression tree modelling to investigate response shift patterns in dentine hypersensitivity. BMC Med Res Methodol, 17: 120-130.

Matsuka Y, Yatan H, Kuboki T. 2008. Temporomandibular disorders in the adult population. J Am Dent Assoc, 24: 158 162.

McNamara Jr JA, Seligman DA, Okeson JP. 1995 Occlusion, orthodontic treatment, and temporomandibular disorders: A review. J Orofacial Pain, 9: 73-90.

Melchior MO, Mazzetto MO, Felício CM. 2012. Temporomandibular disorders and parafunctional oral habits: An anamnestic study. Dental Press J Orthod, 17: 8389.

Michelotti A, Cioffi I, Festa P, Scala G, Farella. 2010. Ora parafunctions as risk factors for diagnostic TMD subgroups. J Oral Rehabil, 37: 157-162.

Mottaghi A, Razavi SM, Pozveh EZ, Jahangirmoghaddam M. 2011. Assessment of the relationship between stress and temporomandibular joint disorder in female students before university entrance exam (Konkour exam). Dent Res J (Isfahan), 8: 76-79.

Mujakperuo HR, Watson M, Morrison R, Macfarlane VT. 2010. Pharmacological interventions for pain in patients with temporomandibular disorders. Cochrane Database Syst Rev, 10: CD004715.

Patel RB, Mathur MB, Gould M, Uyeki TM, Bhattacharya J, Xiao Y, Khazeni N. 2014. Demographic and clinical predictors of mortality from highly pathogenic avian influenza A (H5N1) virus infection: CART analysis of international cases. PLoS One. 25(9): e91630.

Pullinger AG, Seligman DA. 2000. Quantification and validation of predictive values of occlusal variables in temporomandibular disorders using a multifactorial analysis. J Prosthet Dent, 83: 66-75.

Pullinger AG, Seligman DA, Gornbein JA. 1993. A multiple regression analysis of risk and relative odds of temporomandibular disorders as a function of common occlusal features. J Dent Res, 72: 968-979.

Rosenfeld B, Lewis C. 2005. Assessing violence risk in stalking cases: A regression tree approach. Law Hum Behav, 29: 343 
357.

Sato F, Kino K, Sugisaki M, Haketa A, Amemori Y, Ishikawa T, Shibuya $T$, Amagasa $T$, Shibuya $T$, Tanabe $H$, Yoda $T$, Sakamoto I, Omura K, Miyaoka H. 2006. Teeth contacting habit as a contributing factor to chronic pain in patients with temporomandibular disorders. J Med Dent Sci, 53: 103-109.

Scrivani SJ, Keith DA, Kaban LB. 2008. Temporomandibular disorders. N Engl J Med, 359: 2693-2705.

Tagkli A, Paschalidi P, Katsadouris A, Tsolakis IA. 2017. Relationship between orthodontics and temporomandibular disorders. Balk J Dent Med, 21: 127-132.

Uhac I, Kovac Z, Valentic-Peruzovic M, Juretić M, Moro LJ, Grzić R. 2003. The influence of war stress on the prevalence of signs and symptoms of temporomandibular disorders. J Oral Rehabil, 30: 211-217.

Van den Broek AM, Feenstra L, De Baat C. 2007. A review of the current literature on aetiology and measurement methods of halitosis. J Dent, 35: 627-635.
Vanderas AP, Papagiannoulis L. 2002. Multifactorial analysis of the aetiology of craniomandibular dysfunction in children. Int J Paediatr Dent, 12: 336-346

Vayssières MP, Plant RE, Allen-Diaz BH. 2000. Classification trees: An alternative non-parametric approach for predicting species distributions. J Veg Sci, 11: 679-694.

Widmalm SE, Christiansen RL, Gunn SM. 1995. Oral parafunctions as temporomandibular disorder risk factors in children. Cranio, 13: 242-246.

Yamauchi K, Ono Y, Baba K, Ikegami N. 2001. The actual process of rating the global assessment of functioning scale. Compr Psychiatry, 42: 403-409.

Zimmerman RK, Balasubramani GK, Nowalk MP, Eng H, Urbanski L, Jackson ML, Jackson LA, McLean HQ, Belongia EA, Monto AS, Malosh RE, Gaglani M, Clipper L, Flannery B, Wisniewski SR. 2016. Classification and regression tree [CART] analysis to predict influenza in primary care patients. BMC Infect Dis, 16: 503-513. 